Discourse in Filipino Philosophy

\title{
Ang Pagkukuwento bilang \\ Pagbabalik-loob: Pagdalumat sa Halaga ng "Awit ng Matandang Marinero" ni Samuel T. Coleridge sa Talambuhay ni Fr. Roque Ferriols, S.J.
}

Preciosa Regina A. De Joya

\begin{abstract}
At the beginning of Roque Ferriols's autobiography, he remembers the night when he and his father read Samuel $T$. Coleridge's "Rime of the Ancient Mariner," and particularly, how his father translated the English verses to Filipino. This essay explores the significance of Coleridge's long poem to Ferriols's reflection on the purpose and structure of his own autobiographical writing, showing how storytelling is not only a means of atonement towards personal salvation but a process in which reconciliation and re-connection with the community can be achieved. Furthermore, I relate Ferriols's account of the translation of Coleridge's poem to the philosopher's effort at coming to terms with the controversies of the Filipinization movement in the 1970s.
\end{abstract}

Keywords: Coleridge, Ferriols, autobiography, Filipinization movement

\section{Pagdagsa ng Alaala}

$\mathrm{N}$

oong taon 2011, sinikap kong makapanayam si Padre Roque Jamias Ferriols, S.J. tungkol sa buhay niya noong panahon ng Ikalawang Pandaigdig na Digmaan. Ngunit sa aking pagkabigo, malubha niyang itinanggi na ibahagi ang kanyang mga karanasan. Hindi inaasahan na kaagarang mauudlot ang aming usapan, pareho kaming natahimik, hindi

(c) 2018 Preciosa Regina A. De Joya

https://www.kritike.org/journal/issue 22/de joya june2018.pdf

ISSN 1908-7330

$(c c))$ BY-NC-ND 


\section{P. DE JOYA 57}

mawari kung paano magpapatuloy. Sa isip ko: Paano na? Pinangarap ko pa naman na magiging isang ekslusibong pagsisiwalat ang interbyu ng mga lihim na pangyayari sa buhay ng noon 87-taong gulang na Heswitang pilosopo. Sa halip, parang pinagsarahan ako ng pinto, pinagbawalang makapasok, at ang masaklap, wala ito sa mga binalak ko.

Habang iniisip ko ito, si Ferriols naman ay walang imik na nakaupo, parang guwardiyang hindi matinag, nagbabantay nang walang makapasok sa yungib ng kanyang alaala. Naglakas-loob akong tanungin ang dahilan ng kanyang pagtanggi, nagbakasakali na sa kanyang pagsagot, mahikayat siyang tuloy-tuluyan nang magkuwento. Bagaman muling nabigo ang aking plano, ipinaliwanag sa akin ni Ferriols ang hirap na kanyang dinaranas kapag inaalala niya ang mga panahon na iyon. May mga kaibigan siyang nasawi sa digmaan, at sa ilan naman sa kanila na nakaligtas, nakita niya ang patuloy na panghihina ng kanilang katawan dulot ng labis na paghihirap sa ilalim ng mga Hapon. Aniya, may mga bagay na mahirap o masakit kapag inaalala. Pagkatapos niyang sabihin ito, nanahimik siyang muli. Ngunit mapapansin na patuloy na nangungusap ang kanyang mga mata, malalim at mapanimdim, habang nakatingin sa malayo. Para bagang sa sandali na pinasya niyang huwag ungkatin ang nakaraan, dali-dali siyang inanod at tinangay ng kanyang mga alaala. Mababakasan ang lungkot at bigat sa kanyang mukha, para bagang may aninong biglang dumapo sa kanyang malay-tao.

Noong 2012, isang taon makalipas ang "hindi nagtagumpay" na panayam, nalaman kong may sinimulang blog si Ferriols, na nilalaman ang kanyang mga karanasan noong panahon ng giyera. Dahil sa kanyang karamdamang Parkinson's, malaki ang naitulong ng mga nars at tagapangalaga sa Jesuit infirmary upang maitala ang mga kuwento na kanyang idinikta. Ito, kalaunan, ang naging laman ng talambuhay na inilathala noong 2016, sa pamamatnugot ni Leovino Garcia.

Laking tuwa ko na sa wakas ibinahagi ni Ferriols ang kanyang mga karanasan. Kamakailan, tinanong ko siya kung bakit nagbago ang isip niya. Tulad nang mapapansin sa istilo ng pagsasalaysay ng may-akda, simple, banayad, at walang kapritsyo o pagmamalabis na pagdadrama: aniya, marami daw ang nagtatanong, kaya ipinasya niya nang ikuwento. Ngunit ngayon na binabasa ko ang kanyang talambuhay, natuklasan ko na nag-iwan pala ng bakas ang aming unang pag-uusap, na kung tutuusin ay lumalampas sa anumang sukat ng tagumpay o kabiguan, at patunay na nauwi ang panayam sa isang pakikipagtagpo. At bakit ko inaangkin ang ganitong palagay? Dahil kung ang diwa ng pakikipagtagpo, ayon sa ipinahihiwatig ng

(C) 2018 Preciosa Regina A. De Joya

https://www.kritike.org/journal/issue 22/de joya june2018.pdf

ISSN 1908-7330 


\section{ANG PAGKUKUWENTO BILANG PAGBABALIK-LOOB}

salitang ugat, ay hango at ukol sa matatagpuan, may hugis at anyo ng darating, na hindi masasabi at hindi inaasahan sapagkat "matutuklasang hindi hinahanap." ${ }^{1}$ At ganyan nga ang nangyari: nang tanggihan ni Ferriols na isiwalat ang kanyang mga alaala, hindi ko inaasahang biglang maharap sa isang masidhing babala: na hindi nararapat at maaring iuwi ang tao sa disenyo't plano ng tagapanayam.

Ngunit paano ko naman nasasabi na nag-iwan ng bakas ang aming pakikipagtagpo? Sapagkat habang nilalakbay ko ang mga salaysay na maingat at masinop na nilatag ng may-akda, at tinatanaw ang mga pirapirasong tanawan ng kanyang nakaraan, mababakasan at mauulinigan pa rin ang pagtanggi noong una ko siyang napanayam. Sapagkat lakarin man ng mambabasa ang buong kapatagan ng mga salita, maingat at tusong inilihim ng may-akda ang hindi masasabing hapdi ng pag-alala, tahimik na nakasukbit at nakakubli sa mga sulok ng kuwento, sa mga biro at tawa, sa kaba at humpay, at buntong-hininga.

\section{Kuwento ng Matandang Manggugulong}

Bilang guro, isa sa mga mahahalagang itinuturo ni Ferriols sa aming mag-aaral ay ang kuwentong "medyo galing" kay Tswang Tsu, ${ }^{2}$ isang pilosopo mula sa Tsina. ${ }^{3}$ Ayon sa kuwento, binalaan ng manggugulong ang hari na ang binabasa niyang libro ng mga yumaong marurunong ay pawang mga labi lamang, at na ang tunay na karunungan ay hindi maipaliliwanag sa salita. Kaya ang daing ng matandang manggugulong na, bagaman sinikap niyang turuan ang kanyang anak, na tunay namang pinakinggan ang kanyang mga salita, siya pa rin, sa edad na pitumpung taon, ang nagpapakapagod. Sa mga salita ni Ferriols, ipinaliwanag ng manggugulong kung bakit naging ganito ang kanyang kapalaran. Aniya,

Sapagkat hindi pa nauunawaan ng anak ko, kung papaanong makagagalaw ang kamay sa pag-itan ng magaan at mabigat. At papaano nga bang makakagalaw

\footnotetext{
${ }^{1}$ Roque Ferriols, Pambungad sa Metapisika (Quezon City: Ateneo de Manila University Office of Research and Publication, 1997), 35.

2 "Tswang Tsu” ang ginamit na baybay ni Ferriols sa pagsasa-Filipino ng pangalan ng pilosopong Tsino na si Chuang Tsu (baybay ayon sa lumang pagsasatitik sa Romano na Wade-Giles).

3 Mahahanap ang kuwento sa Inggles na pinamagatan "Duke Hwan and the Wheelwright" sa libro ni Thomas Merton, The Way of Chuang Tsu, 2nd ed. (New York: New Directions Publishing, 2010).
}

(C) 2018 Preciosa Regina A. De Joya

https://www.kritike.org/journal/issue 22/de joya june2018.pdf

ISSN 1908-7330

(cc) $\mathrm{BY}-\mathrm{NC}-\mathrm{ND}$ 
ang tao ng ganito? Hindi ko kayang sabihin. Kaya ko lamang gawin. Sapagkat, sa paggawa ng gulong, kapag nasabi na ang lahat ng masasabi, ang pinakahalaga ay hindi masasabi. Magagawa lamang. Mauunawaan lamang ng kamay na gumagawa. At kapag nagawa na, lalabas na pinagkataman lamang at pinaglagarian ang lahat ng nasabi. ${ }^{4}$

Mula sa kuwentong ito, mababatid na ang tunay na pag-uunawa ay matatamo lamang sa pamamagitan ng sariling tiyaga at gawa. Ngunit para kay Tswang Tsu, tila may malubhang pagtanggi na bigyan ng anumang halaga ang salita. Bagaman karaniwang isinasalin bilang "daan," ang Tao (o Dao), ${ }^{5}$ sa katunayan, para kay Tswang Tsu, and Tao ay isang misteryo na hindi mabibigyang ngalan, humihigit sa anumang umiiral, kung kaya't hindi maipapahiwatig sa salita, ni sa katahimikan. ${ }^{6}$ At kaya pinagbalaan ng manggugulong ang hari: hindi maiiwasan, dadalhin ng mga matatanda sa kanilang puntod ang lahat ng kanilang nalalaman. ${ }^{7}$ Kahit ang katahimikan ng taong tunay na marunong ay walang kinalaman sa kakulangan o pagkabaog ng salita; hindi nilalayon o sinasadya, ang kanyang kawalangimik ay nanggagaling sa kapanatagan, kalagayang hindi nangangailangan pang magwika.

Paano, kung gayon, babasahin ang talambuhay ni Ferriols, at paano mabibigyang halaga ang mga kuwento na kanyang isinalaysay? Totoo kaya ang babala ni Tswang Tsu, na kahit anong gawing pagsisikap ng tagapakinig o mambabasa, walang tunay na dunong na dumadaloy sa mga salita? Hindi kaya ito mismo ang talagang nangyayari: na habang sabik kong binabasa ang mga kuwento ng buhay ni Ferriols noong panahon ng giyera, hindi maiwasang iuwi ang mga alaala ng may-akda sa pagiging datos, at ang kanyang mga nasawing kaibigan sa pagiging pulos pangalan lamang?

Sa kanyang pagdadalumat, hindi binibigyang pansin ng teologong si Thomas Merton ang masidhing pagtanggi ni Tswang Tsu sa halaga ng salita. Bagkus, ikinakatuwiran ni Merton na, may nananalaytay sa mga salita na "kuwan," na inuugoy ang pag-iisip tungo sa isang landas na hindi napanghahawakan o sinasaklaw ng salita, at habang hindi ito nabibigyang

${ }^{4}$ Ferriols, Pambungad sa Metapisika, 24.

${ }^{5}$ Ginagamit dito ang Tao na siyang baybay na alinsunod sa paraang Wade-Giles sa pagsasatitik sa Romano.

${ }^{6}$ Merton, The Way of Chuang Tsu, 152.

${ }^{7}$ Ibid., 83.

(C) 2018 Preciosa Regina A. De Joya https://www.kritike.org/journal/issue 22/de joya june2018.pdf

ISSN 1908-7330 


\section{ANG PAGKUKUWENTO BILANG PAGBABALIK-LOOB}

dangal, walang saysay ang mga winika. ${ }^{8}$ Samantala, sa pagtalakay ni Ferriols, may tila ungol ng pagsalungat sa paniniwala ng pilosopong Tsino. Sa simula, inaabisuhan ni Ferriols ang kanyang mambabasa na ang kuwento na kanyang isasalaysay ay "medyo galing" kay Tswang Tsu. Bakit medyo lang? Sapagkat mamaya, babaguhin ni Ferriols ang kuwento.

Batay sa maikling salaysay ni Tswang Tsu, nagbigay si Ferriols ng isang palagay sa naging kapalaran ng anak. Ang sabi daw ng mga tao, aniya, nagtatag ang anak ng aklatan, sa paniniwala at tigas-ulong pagpupumilit na sabihin ang pinakamahalaga. Kaya't, ayon kay Ferriols,

buong ingat isinulat [ng anak] sa isang aklat, ang mga sinabi ng kanyang ama. Pagkatapos ay nanawagan daw siya sa lahat ng mga manggugulong na dumalo sila sa isang malaking komperensiya. Maingat niyang isinulat ang bawat kuro-kuro ng bawat dumalo. Nadagdagan ang kanyang aklatan ng sampung makakapal na libro. Pagkatapos, komperensiya ng mga hindi manggugulong na interesado sa gulong: sampu na namang makakapal na libro. Pagkatapos kwestyoneyr sa ganitong bayan, sa ganoong bansa; ispesyal na biyahe, diyan at doon. Parami nang parami ang mga libro. Ang laki laki na ng aklatan. Noong kuwan ay nagsimula raw siyang magsulat ng mga libro upang maihambing ang mga kuro-kuro ni $\mathrm{A}$, sa mga kuro-kuro ni B, ngunit mas orig si $K$, kahit na daig siya ni $D$, kung ehe ang pag-uusapan, at ano kaya ang sinabi ni $\mathrm{W}$, na kinaiinis ni Y... sangkatutak na nota sa paanan ng pahina, sa likod ng libro, sa harapan.... ${ }^{9}$

Ang pambihirang paglalarawang ito ay upang maipabatid sa mambabasa ang kapangyarihan, sabay kahangalan, na maaring kahahantungan ng

${ }^{8}$ Ibid., 82. Sa paliwanag ni Merton, pinahahalagahan ng mundo ang mga libro, sapagkat pinaniniwalaan, na sa pamamagitan nito, napahahalagahan ang Tao. Ngunit iginigiit ni Merton, na ni salita, ni kaisipang napapaloob sa mga salita, ang nagbibigay ng tunay na halaga sa mga libro. May iba pang ("something else") umiiral sa kaisipan na siyang tumutulak tungo sa isang tiyak na direksyon na hindi maipaliwanag o matangkap ng salita. Upang manatiling totoo at tapat sa diwa nitong hindi masabi/masasabi, ginagamit minsan ni Ferriols ang "kuwan," na sinasambit o naririnig sa mga karaniwang usapan bilang kapalit ng salita panandaliang nalimutan o ng ideya na hindi madaling maipaliwanag o mabigyan ngalan.

${ }_{9}^{9}$ Ferriols, Pambungad sa Metapisika, 24.

(C) 2018 Preciosa Regina A. De Joya

https://www.kritike.org/journal/issue 22/de joya june2018.pdf

ISSN 1908-7330

(c) $\mathrm{BY}-\mathrm{NC}-\mathrm{ND}$ 


\section{P. DE JOYA 61}

paggamit ng tao sa salita. Nababagay rin na ipinepresenta ang lahat ng ito bilang sabi-sabi, o kung tawagin, tsismis. Ngunit sa huli, malubhang itinanggi ni Ferriols ang mga haka-hakang ito. Aniya, "Ganyan daw ang naging kapalaran ng anak. Ngunit, hindi ako naniniwala. Sa palagay ko, kuwento lamang iyan na inimbento ng mga masasamang loob."10 "Ang talagang nangyari," dagdag niya, ay nakikinig ang anak sa sinasabi ng kanyang ama, habang nagtatago sa likod ng banaba, at sa sandaling iyon, ay biglang natauhan: sinimulan niyang pagsikapang pagalawin ang kanyang mga kamay, at pagkatapos ng mahabang panahon ng araw-araw na pagsubok, natuto, sa wakas, ang anak na gumawa ng gulong.

Itong bersyon ng kuwento na hinahain ni Ferriols sa atin ay hindi lamang pag-iimbento ng isang mapaglarong isip. Sapagkat ang nakataya dito ay ang panunumbalik ng halaga at dignidad ng salita, na nakita ni Ferriols na madaling ginagawang alanganin ng burarang pagbabasa sa kuwento ng pilosopong Tsino. Kaya, sa kanyang bersyon, dumalaw ang anak sa libingan ng kanyang ama upang ipabatid na, sa kanyang pagsisikap, sa wakas, manggugulong na siya. Ang sabi niya,

Ama, manggugulong na ako. Tama ang sinabi mo. Hindi nga masasabi ang pinakahalaga. Ngunit nagkamali ka yata noong sinabi mong pinagkataman lamang at pinaglagarian ang lahat ng nasabi. Sapagkat kung hindi mo sinabi ang masasabi, hindi ko sana sinubukang gawin ang masasabi. At kung hindi ko sinubukang gawin ang masasabi, hindi sana tinubuan ng paguunawa ang aking kamay. At kung hindi tinubuan ng pag-uunawa ang aking kamay, hindi ko sana napagtiyagaan ang paggawa ng magagaling na gulong. At kung hindi ko napagtiyagaan ang paggawa ng magagaling na gulong, hindi sana ako nakarating sa paggawa ng hindi masasabi. Kailangan ng taong dumaan sa masasabi, bago niya matatamo ang hindi masasabi. Kaya't hindi ko itatapon o igagatong ang mga sinabi mo. ${ }^{11}$

${ }^{10}$ Ibid., 25. Akin ang diin.

${ }^{11} \mathrm{Ibid}$.

(C) 2018 Preciosa Regina A. De Joya https://www.kritike.org/journal/issue 22/de joya june2018.pdf 


\section{ANG PAGKUKUWENTO BILANG PAGBABALIK-LOOB}

Sa wastong paggamit, may kapangyarihan ang salita na nag-uudyok sa tao na kumilos, inuugoy ang pag-iisip patungo sa landas ng paggawa, at kung minsan, sa pagbabalik-loob. Kaya hindi mapagdududahan na sa pagkukuwento ni Ferriols ng kanyang buhay noong digmaan, bagaman mahirap noong una, sinikap niyang sabihin ang masasabi, alang-alang sa lahat na nagtatanong at nais makaalam.

\section{Awit ng Matandang Marinero}

Sinimulan ni Ferriols ang kanyang talambuhay sa isang pagpapaalam. Papunta siya ng nobisyado, at ninais niyang magpaalam sa kanyang kuwarto, kung saan nabasa niya ang napakaraming libro. At sa masusi niyang pagmamasid sa bawat sulok ng silid, naalala niya ang isang gabi na, aniya, nagpaligaya sa kanilang mag-ama. Habang sabay nilang binabasa ang aklat ng "Awit ng Matandang Marinero," isinasalin ng kanyang ama ang mga Ingles na saknong sa Tagalog.

Ingles-Tagalog-Ingles-Tagalog, hali-halili hanggang sa natapos niya ang tula. Kung minsan inihihinto niya ang pagsasalin upang paliwanagin sa akin, halimbawa, kung ano ang "albatross," o kaya kung bakit nagkukumpisal ang marinero sa ermitanyo. Sabi niya, "kahit na mga Protestante sila, meron silang mga ugaling Katoliko." Hatinggabi na noong natapos kaming makipagsapalaran sa mahiwagang marinero. ${ }^{12}$

Ngunit sino ba itong mahiwagang marinero, at ano ang nilalaman ng kanyang kanta?

Ang "Awit ng Matandang Marinero" ay isang mahabang tula na sinulat ni Samuel Taylor Coleridge, isang Ingles na makata, pilosopo, at teologo ng ikalabing-siyam na siglo. Sa simula ng tula, may maririnig na pagdiriwang ng isang kasal. Parating ang mga panauhin, ngunit pinigilan ng matandang marinero ang isa sa kanila. Nais man ng huli na magpatuloy sa pagdiriwang, may kung anong gayuma na pumigil sa kanya, at siya'y napaupong nakinig sa kuwento ng matandang marinero. Isinilaysay ng matandang marinero kung paano niya walang awang pinatay ang isang

${ }^{12}$ Roque Ferriols, Sulyap sa Aking Pinanggalingan, ed. by Leovino Ma. Garcia (Quezon City: Bughaw, Ateneo de Manila University Press, 2016), 6.

(C) 2018 Preciosa Regina A. De Joya

https://www.kritike.org/journal/issue 22/de joya june2018.pdf

ISSN 1908-7330

(c) $\mathrm{BY}-\mathrm{NC}-\mathrm{ND}$ 
albatross, isang ibon na nagdadala ng buenas. Sa pagpaslang nito, hindi nagtagal at biglang nawala ang hangin, at sa loob ng mahabang panahon, nanatiling hindi tumitinag ang bapor sa gitna ng malawak na karagatan. "Tubig, tubig, sa lahat ng dako, ngunit walang ni isang patak na maaring mainom," ang daing ng makatang umaawit. ${ }^{13}$ Sa sobrang pagkauhaw at pagtutuyo ng lalamunan, hindi na makapagsalita ang mga marinero. Hindi nagtagal at dumating ang Babaeng Multo, ang Bangungot ng Buhay-saKamatayan, kasama ang kanyang katambal na si Kamatayan. At sa kanilang larong sugal ng dais, nagwagi ang Babae at napasakanya ang kaluluwa ng matandang marinero, habang ang iba naman ay inangkin ni Kamatayan. Isaisa, nasaksihan ng matandang marinero ang pagpanaw ng kanyang mga kasama, habang sa kanya nakatinging nanunumpa! Dito natauhan ang matandang marinero, na hindi sa kamatayan ang tunay na pagdurusa kundi sa buhay na paulit-ulit na nasasaksihan ang paglipas ng lahat sa kanyang kapaligiran. Hindi ang kanyang mga kasamang marinero kundi siya ang may dala-dala ng tunay na sumpa; sapagkat habang walang tigil niyang naaalala ang kanilang mga mapanuring mata, nakakaramdam siya ng matinding pagiisa.

Sa edad na siyamnapu't dalawang taon, hindi maikakaila na hawig ang kuwento ni Ferriols sa awit ng matandang marinero. Isinasalaysay ng kanyang talambuhay ang unang apat na taon sa Kapisanan ni Hesus. Pumasok siya sa nobisyado noong Mayo, 1941, at noong Disyembre ng taong iyon, sumiklab ang digmaan. Kaya sa buong aklat nito, umaalingasaw ang amoy ng kamatayan. Ngunit hanggang diyan lang kaya ang kahulugan ng mahiwagang marinero sa kuwento ni Ferriols? Dala-dala rin ba ng mananalaysay ang pasan ng isang sumpa?

Sa unang tingin, ang pagbanggit sa kuwento ng mahiwagang marinero ay tila isang ligaw na alaala ng kabataan ng may-akda. Ngunit sa mas mapanuring pagbabasa, maaaring mahinuha na ang kuwento ay hindi lamang halaw na walang isip kundi balangkas na nagbibigay saysay sa buong talambuhay.

\section{Paghahandog}

Bilang paghahandog, inialay ni Ferriols ang kanyang talambuhay sa tatlo niyang kaibigan: kay Gusting, na nagturo sa kanya na manghuli ng

${ }^{13}$ Samuel Taylor Coleridge, Rime of the Ancient Mariner (1908), II:120. Lahat ng isinaling sipi mula sa tula ni Coleridge ay akin.

(C) 2018 Preciosa Regina A. De Joya https://www.kritike.org/journal/issue 22/de joya june2018.pdf

ISSN 1908-7330 


\section{ANG PAGKUKUWENTO BILANG PAGBABALIK-LOOB}

tutubi sa damuhan noong araw sa Sampaloc; kay Jonathan, na nagbigay sa kanya ng libro na mapagsusulatan ng kanyang mga kaisipan; at kay Genesis, na "nagbigay loob" sa kanya na simulan ang pagsusulat. Ngunit mahalagang pansinin na sa kanyang paghahandog, hindi nagsimula ang may-akda sa pagbanggit ng kanilang mga pangalan kundi sa isang kuwento ukol sa pagkamatay at pagpapalibing sa kanyang kaibigang si Gusting.

Sabado noong inilibing namin si Gusting. Namatay siya noong Miyerkules. Noong Martes isang araw lamang ang nalalabi sa kanyang buhay. Noong Lunes dalawang araw. Noong Linggo tatlo. Balang araw parami nang parami ang nalalabi sa kanyang buhay. Paikli nang paikli ang kanyang buhay. Ang mahirap sa tao ay kailangan niyang mamatay.

May mga manok na tutuka-tuka sa aming manukan. Sa bawat saglit nilalapitan sila ni kamatayan. Pero hindi nila alam. Wala silang malay-tao. At mahina ang malay-manok.

Noong bata ako naiinggit ako sa malay-manok sapagkat hindi nila nadarama ang takot sa kamatayan. Ngunit ngayon ikinalulugod kong manirahan sa abottanaw ng aking malay-tao. Sapagkat kaya kong makipagkilala sa sanlibutan at makapagmuni-muni. ${ }^{14}$

Mula sa maikling sanaysay na ito, mahihinuha ang pag-amin ng may-akda na, sa simula, itinuring niya ang kamatayan bilang sumpa, bilang kasumpasumpa: sapagkat hindi lamang isang malubhang suliranin na kailangan ng tao na mamatay, ang masahol pa'y napakatindi ng kanyang kamalayan, na namamalayan niya sa bawat sandali ang nakasisindak na paglapit ng kamatayan. Mabuti pa ang manok, walang kamuwang-muwang.

Sa tula ni Coleridge, hawig ang naging kapalaran ng matandang marinero. At kaya, sa simula, ang awit niya:

Nag-iisa, nag-iisa, puspusang nag-iisa

Nag-iisa sa malawak na malawak na karagatan!

At ni isang santo ang kailanmang naawa

Sa kaluluwa kong lublob sa kasawian

${ }^{14}$ Ferriols, Sulyap, 2.

(C) 2018 Preciosa Regina A. De Joya

https://www.kritike.org/journal/issue 22/de joya june2018.pdf

ISSN 1908-7330 
Napakaraming tao, napakaganda!

At lahat sila'y namatay na nakatihaya

At libu-libong bagay mula sa burak at bulaho

Ang namamalagi, katulad ko.

Alone, alone, all, all alone

Alone on the wide, wide sea!

And never a saint took pity on

My soul in agony

The many men, so beautiful!

And they all died did lie:

And a thousand thousand slimy things

Lived on; and so did I. ${ }^{15}$

Ngunit sa kabila ng lahat ng pagkamatay at pagkabulok, nakita ng matandang marinero ang mga tubig-ahas na, sa kanilang suot na bughaw, makintab na berde, at pelusang itim, lumalangoy sa dagat at nag-iiwan ng guhit ng ginintuang apoy. Sa sandali ng kanyang pagkamangha sa kagandahang nasilayan niya, nakalas ang nakagapos na albatross sa kanyang leeg at "nahulog at lumubog na parang tingga sa dagat."16

Kung para sa matandang marinero, tila napawi ang sumpa nang matunghayan niya ang kagandahan ng kalikasan, gayon din ang pagbabalikloob na ginanap ni Ferriols. Sa pagtanggap at pagpapalugod sa katalagahan at kondisyon ng pagmamalay-tao, na parating alisto't mulat sa darating na katapusan, naging posible ang pasasalamat, pagpapalakas-loob, at pagsusulat, ukol sa pagkakaibigan, sa mga tutubi sa damuhan ng kanyang kabataan, at sa mga nahuling kislap ng alaala.

\section{Pagkukuwento, Pangungumpisal}

Noong gabing iyon na binabasa nilang mag-ama ang kuwento ng matandang marinero, naalala ni Ferriols kung paano panandaliang tumitigil ang kanyang ama sa pagsalin kapag may nais siyang ipaliwanag sa tula. Isa

${ }^{15}$ Coleridge, Ancient Mariner, IV:235.

${ }^{16}$ Ibid., IV:285-290. "And from my neck so free/ The Albatross fell off, and sank/Like lead into the sea."

(C) 2018 Preciosa Regina A. De Joya https://www.kritike.org/journal/issue 22/de joya june2018.pdf

ISSN 1908-7330 


\section{ANG PAGKUKUWENTO BILANG PAGBABALIK-LOOB}

sa mga katangi-tanging binabanggit ng may-akda ay kung paano ipinaliwanag ng kanyang ama kung bakit nangungumpisal ang marinero sa ermitanyo: "Sabi niya, 'Kahit na mga Protestante sila, meron silang mga ugaling Katoliko'"17

Ngunit kung titingnan ang pag-unlad at hugis ng pag-iisip ni Coleridge, bilang tugon sa mga nag-aatigang debate noong panahong iyon, masasabing hindi siya karaniwang Protestante. Anak ng isang Anglikanong pari, madaling nabighani si Coleridge sa itinuturong Unitarianism ng teologong si Joseph Priestley noong nag-aaral pa siya sa Cambridge. Ngunit hindi nagtagal, dulot ng impluwensiya ng Platonismo at Idealismong Aleman, binatikos ni Coleridge ang mga ideya ni Priestley, kasama ang Socinianism at Empiricism ni William Paley, na parehong pinabulaanan ang doktrina ng Santisima Trinidad at Pagsisisi. ${ }^{18}$

Para kay Coleridge, ang pananaw nina Priestley at Paley ay nakaugat sa Empiricism ni John Locke, na inuuwi ang ideya bilang produkto ng ating karanasang pandama, at abstraksyo na nililikha at namamalagi sa isip ng tao. Dahil dito, hindi tuloy mapahalagahan nina Priestley at Paley ang tradisyong Idealismo na hango mula kay Platon, at ang yaman na ibinibigay nito sa Kristiyanong pananaw ukol sa kalikasan ng Diyos. Para sa kanila, nagbunga lamang ito ng katiwalian sa tunay na diwa ng sinaunang Kristiyanismo sa pagbuo ng isang pilosopiyang spirituwal. Ang tunay na pananampalataya sa iisang Diyos Ama ay binubuo ng mga empirikal na pagpapatunay, at kasama dito ang mga himala na nagbibigay ebidensiya na si Hesus ng Nazareno ang Mesiyas ng sangkatauhan. Ngunit wala sa banal na kasulatan ang pagpapakilala sa Diyos bilang tatlong persona, kung kaya ang doktrina ng pagsasakatawang-tao ng banal na espiritu, pati na ang pagsisisi na bahagi ng pananaw ng kaligtasan bilang pakikibahagi sa buhay ng espiritu (nous o logos), ayon kay Priestley, ay mga pagpapalabo dulot ng mga ambisyon ng mga teologo na bigyan ng pilosopikong pundasyon ang Kristiyanismo. ${ }^{19}$

Ngunit nanindigan si Coleridge sa pananaw na ang Diyos ng Kristiyanismo ay hindi nahihiwalay sa mundo kundi buháy at personal, at laganap sa buong daigdig na kanyang nilikha. Nanalig si Coleridge na ang

${ }^{17}$ Ferriols, S.J., Sulyap, 6.

${ }^{18}$ Douglas Hedley, Coleridge, Philosophy and Religion: Aids to Reflection and the Mirror of the Spirit (Cambridge: Cambridge University Press, 2004), 50. Ayon kay Hedley, bagaman naunang umusbong ang Socinianism bilang produkto ng teolohiya ng Repormasyon noong ika16 at 17 na siglo, at ang Unitarianism naman ay hinango mula sa radikal na uri ng Protestantismo ng Socinianism at nabuo lamang noong ika-18 na siglo, kinilala ni Coleridge ang pagkakaisa ng dalawa, bilang puwersang tumutuligsa sa doktrina ng Santisimo Trinidad at Pagsisisi.

${ }^{19} \mathrm{Ibid} ., 54$. 


\section{P. DE JOYA 67}

buong realidad ay hindi lamang mauuwi sa pagiging bagay o res, kundi espirituwal: bahagi at nakikibahagi sa isang Absoluto na buháy na pagmamalay na nakikipag-ugnay at bukal ng kasaganaan at kaganapan ng kabuoang saklaw ng pag-iral. Sa pagpapatunay nito, nagiging mahalaga ang ideya ng Santisimo Trinidad bilang paraan at proseso ng pagpapakilala ng walang hangganang Diyos Ama, na sa pamamagitan ng pagsasakatawan ng Diyos Anak bilang mundong may wakas, bilang Logos, nauunawaan at tumatalab ang Banal sa kamalayang-tao. Sa pamamagitan ni Kristo, nagiging posible ang pagdurusa at pagsisisi bilang gabay sa paglalakbay at panunumbalik ng bawat nilalang tungo sa kaluluwa ng Diyos. ${ }^{20}$

Bilang paggunita sa kuwento ng pagkakatapon ni Cain pagkatapos niyang paslangin ang kanyang kapatid na si Abel, ang pagdurusa at pagsisisi ng matandang marinero ay isang mapanglaw na paglalakbay ng pag-iisa. ${ }^{21}$ Kaya bagaman tila unti-unting napawi ang sumpa nang masilayan niya ang ganda ng kalikasan, natamo lamang nang lubos ng marinero ang ginhawa nang ikinuwento niya ang lahat ng kanyang ginawa sa ermitanyo. At mula noon, sa mga sandaling nanunumbalik ang kanyang pagdurusa, nag-aapoy ang kanyang puso hangga't maisalaysay niya muli ang mga kasindak-sindak na pangyayari ng nakaraan.

Dumaraan akong parang gabi sa iba't ibang lupalop;

Taglay ko ang kakaibang lakas ng pananalita

Sa sandali na mukha niya'y masilayan ko

Batid ko ang taong kailangang pakinggan 'ko

Sa kanya itinuturo ang aking katha.

I pass, like night, from land to land;

I have strange power of speech;

That moment that his face I see,

I know the man that must hear me;

To him my tale I teach. ${ }^{22}$

Sa mga salitang ito, mahihinuha na hindi ganap na naglaho ang sumpa. Patuloy na naglakbay ang matandang marinero, at sa tuwing bumabalik ang apoy sa kanyang dibdib, napipilitan siyang paulit-ulit hanapin ang ginhawa

20 Tingnan ang ibid., 9. Cf. Ibid., 71.

${ }^{21}$ Paul Chi Hun Kim, "Ecotheology and the Idea of Forgiveness in The Rime of the Ancient Mariner," in Literature Compass, 11:2 (2014), 109.

${ }_{22}$ Coleridge, Ancient Mariner, VII: 585-590.

(C) 2018 Preciosa Regina A. De Joya https://www.kritike.org/journal/issue 22/de joya june2018.pdf

ISSN 1908-7330 


\section{ANG PAGKUKUWENTO BILANG PAGBABALIK-LOOB}

sa pagsasalaysay ng kanyang kuwentong-buhay. Para tuloy na sa bawat pagkukuwento, nauulit ang pangungumpisal na unang naganap sa tulong ng mabuting ermitanyo.

Sa pagdadalumat ng antropologong si Michael Jackson, ang "Awit ng Matandang Marinero" ay hindi lamang mauuwi sa isang pangungumpisal na naglalayon ng personal na kaligtasan. Ayon sa kanya, masyadong "Eurocentric" o "ego-centered" ang pananaw na nagsasaad na ang pagsasalaysay ng kuwentong-buhay ay naglalayon lamang ng katarsis at personal na pagpapagaan ng loob. Sapagkat kung ginagawa nga ito ng tao upang mapalaya lamang ang sarili mula sa tanikala ng kahihiyan at pagkakasala, hindi pa rin naipapaliwanag kung bakit may puwersang tumutulak na ulit-ulitin ang pagkukuwento na, sa kaso ng matandang marinero, ginagawa sa alang-alang ng sinumang nais makinig at makaalam. Hindi kaya na, bagaman nagsisimula bilang isang kilos ng indibiduwal ang pagkukuwento, ang tunay na layunin nito ay maibsan ang tao mula sa kanyang pag-iisa at pagkakawalay sa komunidad? Sapagkat hindi maitatanggi na sa pagsisiwalat ng ating pagdurusa bilang tao, natutuklasan kung ano ang nag-uugnay at nagbibigkis sa ating lahat. ${ }^{23}$

Sa pakikinig sa kuwentong-buhay ni Ferriols, maari kaya nating ipalagay na ang pahapyaw niyang pagsambit sa awit ng matandang marinero ay higit sa isang ligaw na alaala? Hindi kaya, sa kabila ng maingat niyang pagkubli at pagkanlong sa awit ng matandang marinero, mauulinigan ang sariling hinaing at pagsusumamo ni Ferriols ng hindi masasabing pagdurusa at hiya, at pagpapangalaga sa dangal ng masidhing pag-iisa na kanyang naramdaman?

\section{Kilusang Pilipinisasyon sa Ateneo (Dekada '70)}

Sa pagbasa ng talambuhay ni Ferriols, hindi maaaring kalimutan ang isa sa mga kontrobersyal na pangyayari na nagdulot sa kanya ng matinding pagdurusa. Noong 1969, sinimulan ni Ferriols na magturo ng pilosopiya sa Filipino. Bagaman kinikilala rin si Ferriols, kasama nina Ramon Reyes at Jose Cruz, S.J., na nagpasimuno ng bagong programa sa pilosopiya noong 1966, na ipinalaganap ang penomenolohiya bilang batayan at metodo sa pamimilosopiya, ang kanyang pagtuturo sa wikang Filipino ay isang makasaysayang hakbang tungo sa pag-uugat ng disiplina sa ating

${ }^{23}$ Michael Jackson, Lifeworlds: Essays in Existential Anthropology (Chicago: University of Chicago Press, 2013), 186-187.

(C) 2018 Preciosa Regina A. De Joya

https://www.kritike.org/journal/issue 22/de joya june2018.pdf

ISSN 1908-7330

(cc) $\mathrm{BY}-\mathrm{NC}-\mathrm{ND}$ 
kabihasnan. Isa sa mga napakahalagang konsepto na binunga ng kanyang pamimilosopiya sa wikang Filipino ay ang ideya ng "Meron," bilang pagsasakatubo ng ideya ng pag-iral hango sa tradisyon ng Tomismo ni Joseph Marèchal.

Dahil sa kanyang pagpapasimuno ng pagtuturo ng pilosopiya sa wikang Filipino, naging bahagi si Ferriols ng kilusang Pilipinisasyon sa Ateneo, na unang sumiklab noong nilimbag ang isang manifesto na pinamagatang "Down From the Hill" (1968), na isinulat ng limang Atenista, kabilang ang makata at aktibistang si Eman Lacaba at ang makata at mamahayag na si Alfredo Salangga. Ipinahayag ng manifesto ang kanilang reklamo laban sa Kapisanan ni Hesus, at sa simbahang Katoliko, na pinapanatili ang, at nakasandal sa, kapangyarihan ng mga elitista, at kaya pati ang oryentasyon ng edukasyon sa Ateneo ay maka-Kanluran, at walang silbi sa pagpapabuti ng bayan. Imbis na nakakatulong sa mga mahihirap, lalong pinatitindi ang pambubusabos na nangangailangang pawiin ng isang rebolusyonaryong pagbabago. ${ }^{24}$ Naging bahagi ng panunumbalik sa mga bagay na "Filipino" ang pag-uusisa, halimbawa, ukol sa pagtuturo ng 18 units ng Ingles sa kurikulum at ang kakulangan sa pagtuturo ng mga kurso ukol sa kasaysayan ng Pilipinas. Iginiit rin ng manifesto na kinakailangang ituro ng mga Filipino mismo ang mga mahahalagang kurso tulad ng ekonomiya, kasaysayan, at agham pampulitika, at hindi dapat ipinagkakatiwala sa mga Amerikanong guro.

Nakiisa si Ferriols sa hinaing ng mga mag-aaral, at ipinaliwanag sa mga kritiko ng "Down From the Hill” na kung hinihingi man nilang baguhin ang oryentasyon ng kurikulum, at na ituro ng mga Pilipinong guro ang mga kritikal na kurso, ito ay dahil, aniya, hindi kaya ng isang lumaki sa banyagang lupain na maging sensitibo sa mga krisis na hinaharap ng ating bayan, o sa angking yaman ng ating kultura. Hindi ito panlalait sa isang taga-labas, kundi katotohanan lamang ukol sa kanyang katayuan bilang dayuhan. ${ }^{25}$

Sa isang panayam, sinabi ni Ferriols na may iilang Amerikanong Heswitang nagalit at nagbintang sa kanya ng pagsulsol sa mga mag-aaral na labanan sila. Sa pakiwari niya, ito ay nagdulot ng sama ng loob sapagkat inakala nila na politikal ang kanyang ipinaglalaban. Sa kanya, malinaw na hindi politikal, tulad ng "Down From the Hill," ang pinag-uugatan ng kanyang Pilipinisasyon, kundi teolohikal, isang pagsisikap na isakatuparan

${ }^{24}$ Para sa mga detalye, paki tingnan ang Preciosa Regina de Joya, "In Search of Filipino Philosophy" (Ph.D. Dissertation, National University of Singapore, 2014).

${ }^{25}$ Ramon V. Puno and Vicente A. Cabanero, "A Call for Cultural Realism," in The Guidon (11 December 1968), 3.

(C) 2018 Preciosa Regina A. De Joya https://www.kritike.org/journal/issue 22/de joya june2018.pdf

ISSN 1908-7330 


\section{ANG PAGKUKUWENTO BILANG PAGBABALIK-LOOB}

ang pluralismong itinataguyod ang kaganapan ni Kristo sa iba't ibang kultura. Ito, ayon kay Ferriols, ay nakasaad sa isang tekstong isinulat niya noong 1955, na pinamagatang, "Theological Aspects of Cultural Adaptation." ${ }^{26}$ Malinaw niyang ipinaliwang ito sa isang interbyu sa Guidon noong 1968, ngunit, aniya, hindi ito sinama sa artikulong nilimbag. Aniya, kung naunawaan ng mga Amerikanong Heswita na teolohikal ang batayan ng kanyang pilipinsasyon, siguro mas naunawaan nila kung ano talaga ang kanyang hinahangad. Kaya ipinaliwanag sa akin muli ni Ferriols:

Hindi ko nilalabanan ang mga Amerikano. Nag-udyok ako na magpaka-Filipino. At kung ikaw ay magpakaFilipino, may mga gawaing Amerikano na hindi ka maaring sumang-ayon. Hindi sapagkat ayaw mo Amerikano, pero sapagkat gusto mo ng Filipino. (Panayam, 2009).

Bilang tugon sa tampo ng mga Amerikanong madaling nag-akalang walang pagpapahalaga sa kanilang nagawa, sinikap ni Ferriols noong magulong panahon na iyon na ipaliwanag ang kasalimuotan ng sitwasyon, na hindi maaring iuwi sa simpleng pagtanggi o pagkamuhi sa dayuhan.

Ang atitud ko ay meron akong appreciation sa kanilang ginawa, pero yung kanilang ginagawa ay magiging buo, magiging mas buo.... kagaya ng sinabi ko sa isang meeting namin[g mga Heswita]. Sabi ko, the Americans have done a great deal for the Philippines, but they have to do something harder. To let the Filipinos become really Filipinos. To do that, the Americans have to give up some of their American ways. The Americans can do it because... kasi may slogan ang mga Amerikano during the war, eh: the difficult we do at once, the impossible takes a little longer. Yun ang isa sa mga slogan ng mga Americans. SEACBEES-isang engineering department ng US Navy ata yon. (Panayam, 2009).

\footnotetext{
${ }^{26}$ Tingnan ang Roque Ferriols, "Theological Aspects of Cultural Appropriation," in Pagdiriwang sa Meron: A Festival of Thought Celebrating Roque J. Ferriols, S.J., ed. by Nemesio S. Que and Agustin Martin G. Rodriguez (Quezon City: Ateneo de Manila University Office of Research and Publications, 1997), 265-83.

(C) 2018 Preciosa Regina A. De Joya

https://www.kritike.org/journal/issue 22/de joya june2018.pdf

ISSN 1908-7330

(cc) $\mathrm{BY}-\mathrm{NC}-\mathrm{ND}$
} 


\section{Hali-halili}

May mga nagsasabi na nahirapan si Ferriols dahil sa kontrobersyang ito, at may iba pang nagsasabi na dahil dito, nalulong siya sa pag-iinom at nangailangang dumaan ng psychotherapy. Ngunit hindi maikakaila na, habang binabasa ang kanyang talambuhay, isinasalaysay ng may-akda, hindi ang alitan at pagkakaiba kundi, ang pagkakaisa at pagtutulungan ng mga Amerikanto't mga Filipino noong panahon ng digmaan. Isa kaya itong pagbabalik-loob, pagpapahayag ng may-akda ng pagsisisi sa nangyaring kaguluhan noong dekada '70? O isa kayang paraan ng pagwawasto ng mga maling akala't pagtutuwid ng baluktot na pag-uunawa?

Sa buong talambuhay, puspusang pinasasalamatan ng may-akda ang kanyang mga guro, Amerikano't Filipino, na hali-haliling nagtulong-tulong na gawing posible ang buhay, ang pag-asa at pagtuto, "ang pag-aaral ng Griyego at Latin at pagtanim ng pechay at kamote ..." 27 sa kabila, at sa gitna, ng kaguluhan. Kasama rin ang mga kuwento ng samahan at pakikiramay, sa pakikibahagi sa lungkot at kawalan na dulot ng katangahang pinairal ng digmaan, at mga kaibigang kasama at hindi maiwan sa gitna ng panganib at karahasan. Sa pagkukuwento ni Ferriols sa mga kaganapan noong madilim at magulong panahong iyon, mauulinigan ang awit ng matandang marinero: na tila nagsasabi na sa ating pagdudurusa, mahaba man ang paglalakbay at pag-iisa, natutuklasan rin sa huli ang pagkakaisa at pagbubuklod nating lahat bilang tao-na tulad ng mga Katoliko't Protestante, masasabi rin na kahit Amerikano sila, may mga ugali rin silang Filipino.

Department of Philosophy, Ateneo de Manila University, Philippines

\section{References}

Coleridge, Samuel Taylor. "Rime of the Ancient Mariner." Mula sa Coleridge's Ancient Mariner and Select Poems. Pat. Frederick H. Sykes. Scribner English Classics, 1908. Kindle Edition.

De Joya, Preciosa Regina, "In Search of Filipino Philosophy," (Ph.D. Dissertation, National University of Singapore, 2014).

Ferriols, Roque, Pambungad sa Metapisika (Quezon City: Ateneo de Manila University Office of Research and Publication, 1997).

${ }^{27}$ Ferriols, Sulyap, 43.

(C) 2018 Preciosa Regina A. De Joya https://www.kritike.org/journal/issue 22/de joya june2018.pdf

ISSN 1908-7330 


\section{ANG PAGKUKUWENTO BILANG PAGBABALIK-LOOB}

Sulyap sa Aking Pinanggalingan. Pat. Leovino Ma. Garcia (Quezon City: Bugaw, Ateneo de Manila University Press, 2016).

"Theological Aspects of Cultural Appropriation," in Pagdiriwang sa Meron: A Festival of Thought Celebrating Roque J. Ferriols, S.J., ed. by Nemesio S. Que and Agustin Martin G. Rodriguez (Quezon City: Ateneo de Manila University Office of Research and Publication, 1997).

Hedley, Douglas, Coleridge, Philosophy and Religion: Aids to Reflection and the Mirror of the Spirit (Cambridge: Cambridge University Press, 2004).

Kim, Paul Chi Hun, "Ecotheology and the Idea of Forgiveness in The Rime of the Ancient Mariner," Mula sa Literature Compass, 11:2 (2014).

Jackson, Michael, Lifeworlds: Essay in Existential Anthropology (Chicago: University of Chicago Press, 2013).

Merton, Thomas, The Way of Chuang Tsu, $2^{\text {nd }}$ ed. (New York: New Directions Publishing, 2010).

Puno, Ramon V. and Vincente A. Cabanero, "A Call for Cultural Realism," mula sa The Guidon (11 December 1968).

(C) 2018 Preciosa Regina A. De Joya

https://www.kritike.org/journal/issue 22/de joya june2018.pdf

ISSN 1908-7330 